Gastroenterologia. 1954;82:176

\title{
Erwiderung zu den Bemerkungen von Broicher
}

O.

Otto

Roth

Winterthur

Meiner Erfahrung nach muß an der Tatsache festgehalten werden, daß ein Ulcus callosum weder röntgenologísch noch gastroskopísch mit Sicherheit ausgeschlossen werden kann.

Für die Beurteilung der Wirksamkeit von Robuden wurden in der Versuchsanordnung Broichers ausschließlich schwere, chronisch rezi-divierende Ulzera mit langer Anamnese herangezogen, die erfahrungs-gemäß therapeutisch sehr schwer beeinflußbar sind. 26 solcher Fälle sprachen auf Robuden nicht an. Es erstaunt mich aber, erst den Bemerkungen des Autors zu entnehmen, daß alle diese Ulzera nach einer Zeit von 12 Wochen doch noch ausheilten. In der Originalarbeit findet man, aus mir völlig unbegreiflichen Gründen, kein Wort darüber.

Angesichts dieses Sachverhaltes kann es sich in meinen Ausfüh-rungen nicht um Irrtümer handeln, sondern um einen Interpretations-versuch irreführender und leider sehr unzulänglicher Darlegungen des Autors. Zwei Röntgenaufnahmen recht fraglichen Wertes und zwei Beispiele des Krankheitsablaufes können darüber gewiß nicht hinwegtäuschen.

Der Wert eines Therapeutikums ist in der Klinik leichter zu er-mitteln als ambulant. Im Falle von Ulkuskrankheit aber sind die Resultate ambulanter Behandlung entschieden aufschlußreicher. Wenn die Erfolgsquote der poliklinischen Robudenbehandlung mit derjenigen der klinischen durchaus Schritt halt, so ist dies als ein wesentlicher Vorteil von Robuden zu bewerten. Denn die Behandlung des Ulcus ist und bleibt, aus verschiedenen sehr gewichtigen Gründen, in erster Linie eine ambulante. 